\title{
Calorimetric Studies of the Polynucleotide Analog Poly(-)2-[2-(thymin-1-yl) propanamido]propenoic Acid
}

\author{
R. ZAND,* Z. Z. JIANG, and C. G. OVERBERGER, Macromolecular \\ Research Center and Biophysics Research Division, Institute of \\ Science and Technology, Department of Biological Chemistry and \\ Department of Chemistry, University of Michigan, Ann Arbor, \\ Michigan 48109
}

\begin{abstract}
Synopsis
The thermally elicited structural and conformational transitions of the polynucleotide analog, poly(-)-2-[2-(thymin-1-yl)propanamido]propenoic acid, P(-)TDHA, have been studied using differential scanning calorimetry (DSC), differential thermal analysis (DTA), and thermogravmetric analysis (TGA). The differential scanning calorimetry curves obtained on solid P(-)TOHA samples exhibited five distinct transitions. The transition occurring at $50^{\circ} \mathrm{C}$ is attributed to a disruption of interactions involving thymine-thymine stacking. In contrast the transition observed at $83^{\circ} \mathrm{C}$ is attributed to a hydrogenbonding interaction involving the thymine residues, whereas the transition occurring at $110^{\circ} \mathrm{C}$ is assigned to hydrogen bonding of the carboxylic acid side-chain groups. The transitions observed at 50,83 , and $110^{\circ} \mathrm{C}$ are reversible if the heated and quenched sample is allowed to equilibrate in an atmosphere of high humidity. The transition occurring at $127^{\circ} \mathrm{C}$ is viewed as a structural rearrangement of the polymer backbone that does not involve the participation of water molecules. The transition observed at a temperature above $197^{\circ} \mathrm{C}$ is attributed to a structural modification of the polymer resulting from decomposition. Solutions of $\mathrm{P}(-) \mathrm{TDHA}$ in $0.1 \mathrm{M}$ phosphate buffer at $\mathrm{pH}$ 7.05 showed only a single transition at $50^{\circ} \mathrm{C}$, which is in accord with an observed transition in the solid state assigned to the disruption of base-stacking interactions. The average enthalpy for the transition at $50^{\circ} \mathrm{C}$ was $0.92 \mathrm{cal} / \mathrm{g}$ in the solid state and 1.08 $\mathrm{cal} / \mathrm{g}$ for the solution, which provides additional support for the assignment.
\end{abstract}

\section{INTRODUCTION}

Synthetic analogs of DNA and RNA offer simple model systems for the investigation of factors that influence the structure of such molecules, as well as the reported bioactivity of these materials. Although a number of analog systems have been reported, ${ }^{1-4}$ most have been hydrophobic and lacking in chirality. This is in contrast to the natural nucleic acids that are very hydrophilic and contain centers of asymmetry in the carbohydrate moiety. Since several analog systems have been reported that possess antiviral- and interferon-inducing activity, ${ }^{5,6,7}$ the role of secondary and tertiary structure in conferring bio-

\footnotetext{
* To whom correspondence should be addressed.
} 
logical activity becomes a significant factor to be investigated. In the group of synthetic homopolynucleotides, it has been established that several of these macromolecular systems do not have random-coil structures but have ordered conformations with specific orientations that can be assigned to the polymer backbone and to the side-chain groups. $8,9,10$

This study has addressed the question of the presence of ordered structure in a polymer of dehydroalanine that has a pendant side chain containing a thymine group attached to a chiral carbon atom, I.

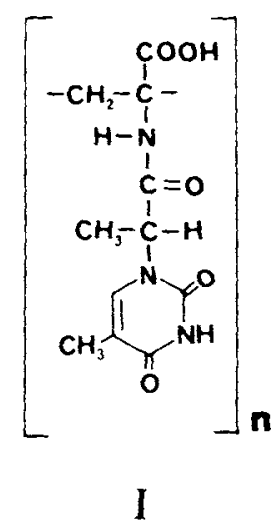

Structure I. Poly(-)2-[2-(thymin-1-yl)propanamido] propenoic acid.

The temperature-dependent transitions observed have been tentatively assigned to specific types of structural changes in the molecule, thereby permitting an assessment of base-base interaction and backbone stability in the polymer chain.

\section{EXPERIMENTAL}

Polymer samples were prepared and purified as described previously. ${ }^{11}$ Sample 3065 had an intrinsic viscosity of $0.86 \mathrm{dL} / \mathrm{g}$ while sample 4046 had an intrinsic viscosity of $0.87 \mathrm{dL} / \mathrm{g}$. After drying in a vacuum oven at $90^{\circ} \mathrm{C}$ the polymers still retained trace amounts of water.

The differential scanning calorimeter used in this study was a Perkin-Elmer model DSC-2C. Data acquisition was by a Perkin-Elmer thermal analysis data station. Sealed aluminum pans were used to contain both solid and liquid samples; typically 1 to $12 \mathrm{mg}$ of sample was used. The sensitivity of the instrument was adjusted to $1 \mu \mathrm{cal} / \mathrm{s}$ for the solid samples and $0.3 \mu \mathrm{cal} / \mathrm{s}$ for the samples dissolved in buffer. The solutions were prepared in $0.1 M$ phosphate buffer, $\mathrm{pH} 7.05$. The temperature and differential heats were calibrated with standard indium and tin references.

The differential thermal analyses curves were obtained on a DuPont 900 differential thermal analyzer. A typical heating rate was $20^{\circ} \mathrm{C}$ per 
minute. The thermogravimetric analyses were obtained on a DuPont 950 thermogravimetric analyzer.

\section{RESULTS}

The DSC curves obtained on solid samples of P(-)TDHA are shown in Fig. 1. The insert curve of Fig. 1 covers the temperature range from 290 to $600 \mathrm{~K}\left(17\right.$ to $\left.327^{\circ} \mathrm{C}\right)$, and the endothermic transition appears as a broad band with a second transition at $400 \mathrm{~K}$; at $510 \mathrm{~K}$ the curve goes off-scale. Upon cooling and rescanning, the broad endotherm and the second transition at $400 \mathrm{~K}$ are no longer observed; however, the rise occurs at $490 \mathrm{~K}$, which is about $20^{\circ}$ lower than in the original scan. With a larger amount of polymer and an expanded scale, one obtains a typical curve as shown in Fig. 1. Here the broad endothermic transition has been resolved into three distinct transitions with onsets for respective transitions occurring at $323 \mathrm{~K}\left(50^{\circ} \mathrm{C}\right), 356 \mathrm{~K}\left(83^{\circ} \mathrm{C}\right)$, and $383 \mathrm{~K}\left(110^{\circ} \mathrm{C}\right)$.

The TGA curve, Fig. 2 , shows no change in the weight of the polymer in this temperature range until $220^{\circ} \mathrm{C}$. Therefore, the endothermic peaks in the DSC curves must reflect nondestructive structural alterations. The transition at $127^{\circ} \mathrm{C}$ is also seen in the DTA curve. Since these specific endotherms are reversible after reequilibration in an atmosphere of high humidity, we are led to infer that part of this change must involve a loss of interaction with water molecules. The TGA curves show a slight downward trend with temperature from 20

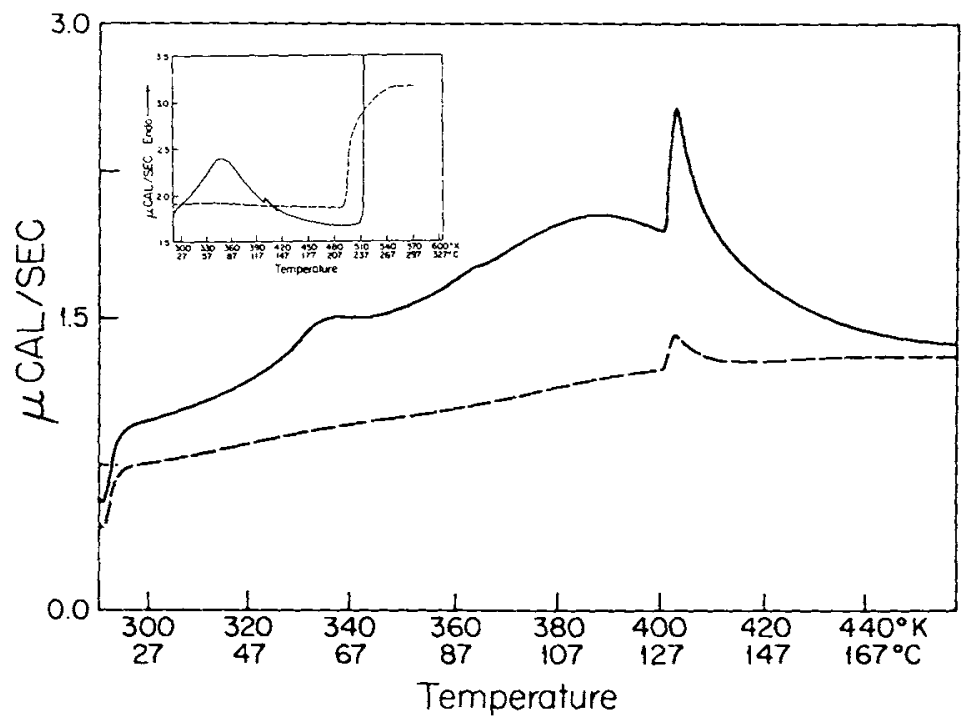

Fig. 1. DSC curve of solid P(-)TDHA plotted on an expanded temperature scale. The curve was obtained from $10 \mathrm{mg}$ of polymer. Insert is the curve obtained from only 5 $\mathrm{mg}$ of polymer. 


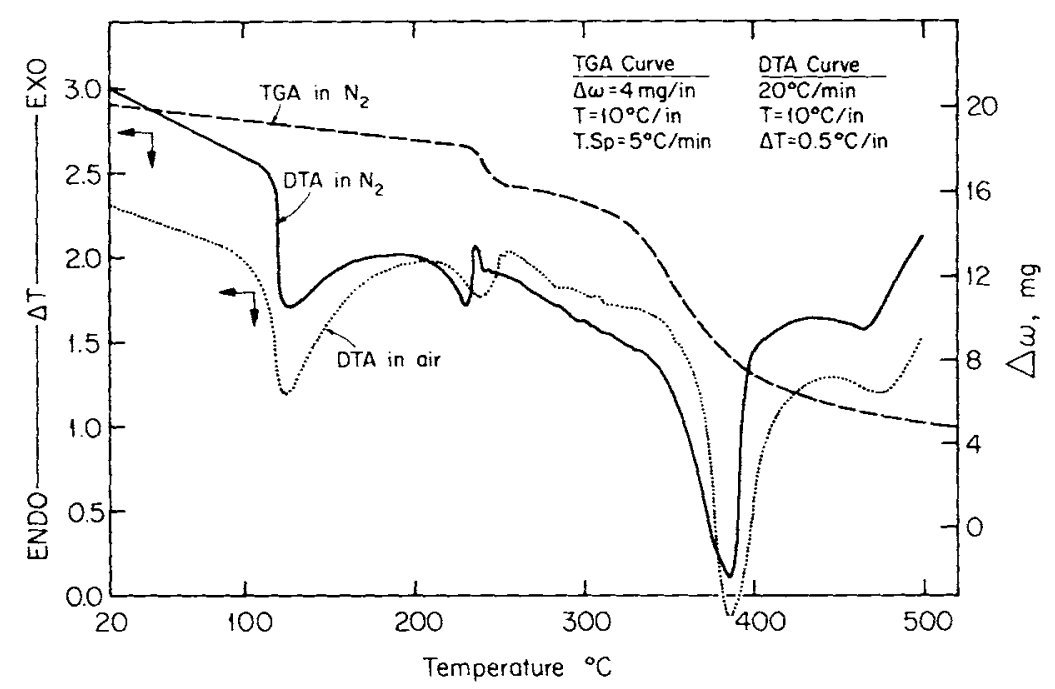

Fig. 2. DTA and TGA curves of P(-)TDHA showing minimal weight loss of the TGA curve until $220^{\circ} \mathrm{C}$. The observed transition above $220^{\circ} \mathrm{C}$ in the DTA curve is paralleled by weight loss in the TGA curve.

to $230^{\circ} \mathrm{C}$. In the region of $230-240^{\circ} \mathrm{C}$, there is a transition reflecting a change in the local structure with a small loss in weight.

The transition at $50^{\circ} \mathrm{C}$ is interpreted as arising from a disruption of the base-stacking, thymine-thymine interaction. Some breaking up of hydrogen-bond interactions may also be associated with this transition. The enthalpy for this transition was found to be $0.92 \mathrm{cal} / \mathrm{g}$ which is a reasonable value for such a process. The transition at $110^{\circ} \mathrm{C}$ is much broader and can be considered to be a cooperative process, possibly involving the breakup of carboxyl side-chain hydrogen-bond interactions. The transition observed at $127^{\circ} \mathrm{C}$ is a reversible transition that occurs even in the absence of reequilibration with water vapor, and it probably represents a rearrangement of the backbone structure.

Between 210 and $220^{\circ} \mathrm{C}$ the DTA curves exhibit an endothermic transition followed immediately by an exothermic transition. At this same temperature the TGA curves show a sharp loss in weight and then a plateau for about $40^{\circ} \mathrm{C}$ prior to the onset of a more complete decomposition process. The transition in the region of $210^{\circ} \mathrm{C}$ is viewed as arising from the loss of some molecular component of the polymer. A possible explanation is that this transition reflects a decarboxylation with subsequent structural rearrangement to give a new and more stabile structure. Above $300^{\circ} \mathrm{C}$ the TGA curve shows a dramatic loss in weight; such a change is also seen in the DTA curve. This is interpreted as an indication of the fragmentation of the polymer and of the resulting loss of fragments from the molecule.

The DSC curve obtained on P(-)TDHA in phosphate buffer, $\mathrm{pH} 7.05$ showed only a single endothermic transition at $50^{\circ} \mathrm{C}$, Fig. 3 . This is in 


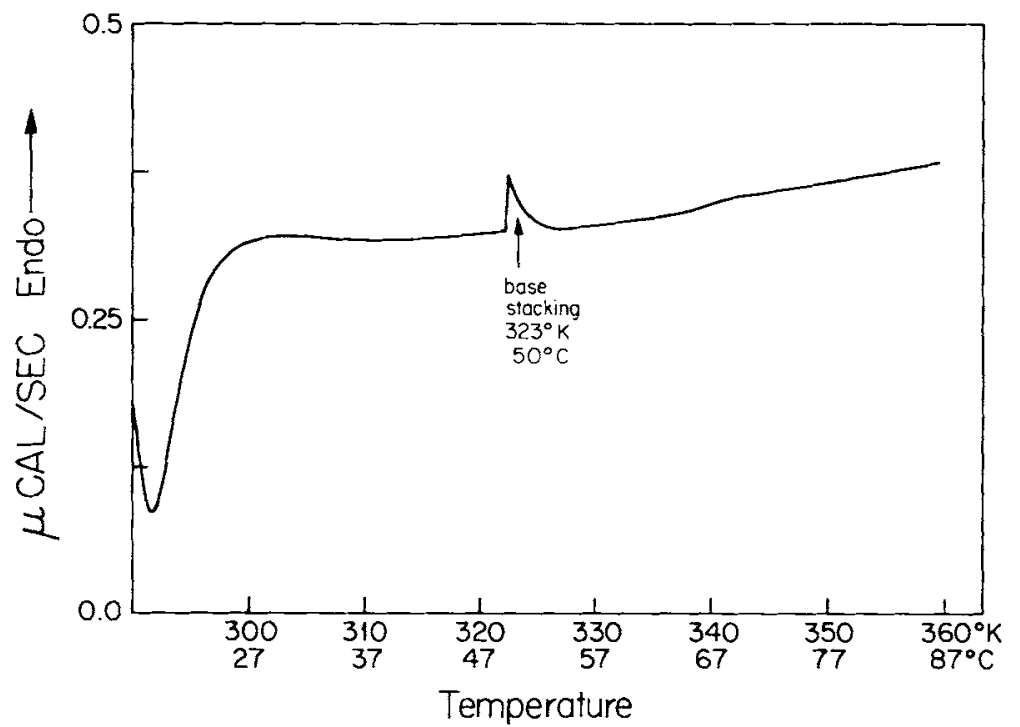

Fig. 3. DSC curve of P(-)TDHA in phosphate buffer $\mathrm{pH} 7.05$ showing only the $50^{\circ} \mathrm{C}$ transition.

accord with our assessment that this transition reflects a disruption of stacked thymine-thymine interaction in the molecule, and that the transition at 88 and $110^{\circ} \mathrm{C}$ present in the solid sample involves hydrogen-bond interactions not broken in aqueous solution. The $\Delta H$ obtained for this transition from the solution data was $1.08 \mathrm{cal} / \mathrm{g}$ which is in good agreement with the value of $0.92 \mathrm{cal} / \mathrm{g}$ obtained for a similar transition observed in DSC curves of the solid sample.

\section{DISCUSSION}

Although DNA preparations have molecular weights of $10^{6}$ daltons and RNAs have molecular weights in the range of $10^{4}-10^{5}$ daltons, while the present analog has a much lower molecular weight, the analogy of P(-)TDHA to the nucleic acids is valid from several points of view. Consideration of the nature of the attached groups demonstrates that, in the nucleic acids, both the pentose and phosphate groups are hydrophilic, whereas in P(-)TDHA the comparable group is the side-chain carboxyl function. The amide group in the side-chain can also interact with water, although less hydrophilic than the carboxyl group. In contrast to the purine bases, thymine reportedly shows no particular preference for water over ethanol as a solvent. ${ }^{12}$ This has been viewed as an indication that thymine participates in a strong hydrogen-bonding interaction with these solvents. Our calorimetric results support such an assessment of thymine-water (solvent) interaction. The transitions observed at 83 and $110^{\circ} \mathrm{C}$ in the DSC experiments on the solid polymer are viewed as arising from the disruption 
of hydrogen bonds. These may be predominantly carboxyl group $-\mathrm{H}_{2} \mathrm{O}$ hydrogen bonds plus a contribution from the thymine $-\mathrm{H}_{2} \mathrm{O}$ hydrogen bonding. The lower transition at $50^{\circ} \mathrm{C}$ is more reasonably assigned to the disruption of base stacking in which the stacked thymine may also be involved in hydrogen bonds with the solvent. We believe that the $\mathrm{H}_{2} \mathrm{O}$-independent transition observed at $127^{\circ} \mathrm{C}$ is the result of conformational changes of the polymer backbone. The transition at $197^{\circ} \mathrm{C}$ is attributed to polymer degradation.

The current assessment of nucleic acid structure and base-base interactions considers two types of interaction as the most important in influencing structure: A hydrogen-bonding or in-plane interaction and a base-stacking or vertical interaction. DeVoe and Tinoco ${ }^{13}$ have shown that the van der Waals-London forces that must be operative for vertically stacked bases ${ }^{14}$ also function on the in-plane interactions between bases. In view of the strong thymine-water hydrogen-bonding interaction that would deter the formation of in-plane thyminethymine interactions, the assignment of the $50^{\circ} \mathrm{C}$ transition as a baseunstacking transition is the most reasonable interpretation of our calorimetric data. The transitions at 83 and $110^{\circ} \mathrm{C}$ reversible in the presence of water are then logically attributable to the disruption of $\mathrm{H}_{2} \mathrm{O}$-analog molecule hydrogen-bond interactions.

Such interactions could provide stabilization of the folded polymer structure via the interaction of solvent, and hydrogen-bond formation, with the carboxyl groups of the polymer. It is not necessary that the maximum possible number of hydrogen bonds be present in order to arrive at a thermodynamically stable configuration relative to the random or unfolded structure. ${ }^{12}$ Stability is accomplished by a summation of all contributing interaction forces.

The calorimetric data obtained on this polymer show that the unfolding of $\mathrm{P}(-) \mathrm{TDHA}$ is not a simple two-state process such as

$$
\mathrm{P}(-) \mathrm{TDHA}_{\text {(ordered) }} \rightleftharpoons \mathrm{P}(-) \mathrm{TDHA}_{\text {(disordered) }}
$$

The presence of clearly discernible transitions in both the solid polymer and in solutions of the polymer indicates that the unfolding occurs by a multistep mechanism involving intermediate structures. The broad temperature-dependence of the transition centered at ca. $110^{\circ} \mathrm{C}$ may be indicative of cooperative behavior for this structural change.

This calorimetric characterization of P(-)TDHA has demonstrated that the thermal behavior carries a close parallel to the thermal behavior of DNA and RNA, and that analogs based on the polydehydroalanine backbone are valuable model systems for the natural polymers. Furthermore, the results obtained provide compelling evidence for the presence of organized structure in this polymer both in solution and in the solid state, although the data do not permit a discrimination of the possible kinds of thymine-thymine interaction. 


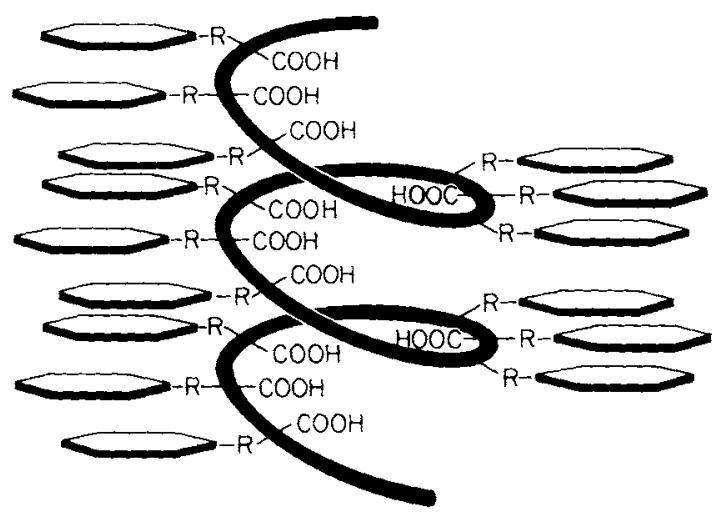

II

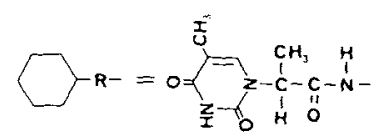

Structure II. Helical conformation of P(-)TDHA backbone and base stacking of the sidechain thymine base.

Three possible models consistent with the interactions we believe to be present are shown in structures II and III. In structure II the dehydroalanine backbone is coiled into a helix and the thymine residues are stacked much like the steps of a spiral staircase. Intramolecular hydrogen bonds between carboxylic acid groups and hydrogen bonds of the side-chain amide groups are controlled by the chain geometry in a given environment. The models in structures III(A) and

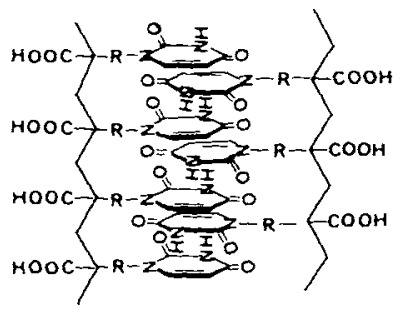

A

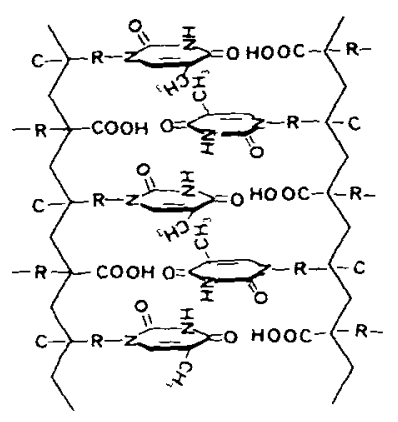

B

III

Structure III. Extended structure of P(-)TDHA and two possible modes of base stacking. The thymine residues are on the same side of the backbone (isotactic) (A), whereas the thymine residues alternate with the carboxyl group for the same side of the backbone (syndiotactic) chain (B). Note that each structure gives rise to hydrogen bonds from different group pairings. 
III(B) in which the dehydroalanine backbone has an extended configuration require that thymine stacking occurs by intercalation. In structure III(A) the thymine groups are on the same side of the chain and give rise to an isotactic structure. In III(B) the thymine groups alternate with the carboxyl group for the same side of the chain and give rise to a syndiotactic configuration. In these structures the base-stacking and hydrogen-bond interactions can arise either intramolecularly from the folding of the linear chain segments upon itself or from intermolecular alignment of two chains. If the polynucleotides are taken as a precedent for modeling the structure of P(-)TDHA, one might prefer structure II on the grounds that it could accommodate the data as a single chain. In contrast to this, III(A) and III(B) would require that the chain either be folded upon itself or that two chains align themselves properly in order to achieve thymine stacking.

\section{References}

1. Jones, A. S. (1979) Int. J. Biol. Macromol. 1, 194-207.

2. Takemoto, K. \& Inaki, Y. (1981) Adv. Polym. Sci. 41, 1-51.

3. Kaye, H. \& Chou, H. J. (1975) J. Polym. Sci., Polym. Phys. Ed. 13, 477-492.

4. Pitha, J. (1977) Polymer 18, 425-430.

5. Stebbing, N. (1979) Pharm. Ther. 6, 291-332.

6. Pitha, J. (1980) in Anionic Polymeric Drugs, Donaruma, L. G., Ottenbrite, R. M. \& Vogel, O., Eds., Wiley-Interscience, New York, pp. 277-301.

7. Overberger, C. G., Brandt, K. A., Kikyotani, S. \& Ludwick, A. G. (1981) Polym. Bull. 5, 481-488.

8. Morishma, Y. \& Overberger, C. G. (1979) J. Macromol. Sci.-Chem. A13 5, 573 585 .

9. Overberger, C. G. \& Morishima, Y. (1982) J. Polym. Sci., Chem. Ed. 18, 1247, 1267.

10. Ludwick, A. G. \& Overberger, C. G. (1982) J. Polym. Sci., Chem. Ed. 20, $2123-$ 2138, 2139-2149.

11. Brandt, K. A. \& Overberger, C. G. (1982) Nouv. J. Chim. 6, 673-677.

12. Alvarez, J. \& Biltonen, R. (1973) Biopolymers 12, 1815-1828.

13. DeVoe, H. \& Tinoco, I., Jr. (1962) J. Mol. Biol. 4, 500-517.

14. Michelson, A. M., Ulbrecht, T. L., Emerson, T. R. \& Swan, R. J. (1966) Nature 209, 873-874.

Received July 29, 1985

Accepted November 29, 1985 